doi: $10.15407 /$ ukrbotj74.01.035

\title{
Validation of names of some syntaxa of the Fagetalia sylvaticae from Ukraine
}

\author{
Victor A. ONYSHCHENKO \\ M.G. Kholodny Institute of Botany, National Academy of Sciences of Ukraine \\ 2, Tereshchenkivska Str., Kyiv 01004, Ukraine \\ labzap@ukr.net
}

Onyshchenko V.A. Validation of names of some syntaxa of the Fagetalia sylvaticae from Ukraine. Ukr. Bot. J., 2017, 74(1): 35-36.

M.G. Kholodny Institute of Botany, National Academy of Sciences of Ukraine

2, Tereshchenkivska Str., Kyiv 01004, Ukraine

Abstract. The names of alliance Scillo sibericae-Quercion roboris (deciduous mesic forests of Eastern Europe on rich soils in Forest-Steppe and Steppe regions), associations Stellario holosteae-Fagetum, Euonymo verrucosae-Fagetum, Anthrisco nitidiAceretum pseudoplatani, Tulipo quercetorum-Quercetum roboris and 10 subassociations are validated.

Keywords: Fagetalia sylvaticae, Scillo sibericae-Quercion roboris, syntaxonomy, syntaxon

According to Article 5 of the 3rd edition of the International Code of Phytosocioligical Nomenclature (Weber et al., 2000) "on or after 1.1.2002 the Latin word 'typus' ('holotypus', 'lectotypus', 'neotypus') is to be used expressis verbis for the designation of the type of a syntaxon name". In publications with descriptions of new syntaxa of order Fagetalia sylvaticae Walas 1933 (Ralo, Onyshchenko, 2008; Onyshchenko, 2009), the aforementioned Latin words were omitted, therefore all new syntaxa from these publications need validation. Some syntaxa were invalid for other reasons as well.

\section{All. Asperulo-Fagion Tüxen 1955}

Stellario holosteae-Fagetum sylvaticae Onyshchenko ex Onyshchenko ass. nov. hoc loco. Holotypus: rel. 9 in table 34 (Onyshchenko, 2009: 76-79). Synonym: Stellario holosteae-Fagetum Onyshchenko 2009 (Art. 5, 16). Article 16 states that "on or after 1.1.2002 a typerelevé of an association name must contain the namegiving taxon (taxa)". The type of the Stellario holosteaeFagetum selected in 2009 (table 34, rel. 1) does not contain Stellaria holostea.

Stellario holosteae-Fagetum sylvaticae typicum Onyshchenko ex Onyshchenko subass. nov. hoc loco. Holotypus: rel. 9 in table 34 (Onyshchenko, 2009: 76-79).

Synonym: Stellario holosteae-Fagetum typicum Onyshchenko 2009 (Art. 5).

Stellario holosteae-Fagetum sylvaticae corydaletosum cavae Onyshchenko ex Onyshchenko subass. nov. hoc loco. Holotypus: rel. 10 in table 36 (Onyshchenko, 2009: 84-87). Synonym: Stellario holosteae-Fagetum corydaletosum cavae Onyshchenko 2009 (Art. 5).

(C) V.A. ONYSHCHENKO, 2017

Укр. бот. жмурн., 2017, 74(1)
Stellario holosteae-Fagetum sylvaticae luzuletosum pilosae Onyshchenko ex Onyshchenko subass. nov. hoc loco. Holotypus: rel. 4 in table 33 (Onyshchenko, 2009: 73-76). Synonym: Stellario holosteae-Fagetum luzuletosum pilosae Onyshchenko 2009 (Art. 5).

Euonymo verrucosae-Fagetum sylvaticae Onyshchenko ex Onyshchenko ass. nov. hoc loco. Synonym: Euonymo verrucosae-Fagetum Onyshchenko 2009 (Art. 5). Holotypus: rel. 11 in table 38 (Onyshchenko, 2009: 91-94). This is a relevé of the subassociation Euonymo verrucosae-Fagetum typicum Onyshchenko ex Onyshchenko ass. nov. In earlier publication (Onyshchenko, 2009), the nomenclatural type of the association Euonymo verrucosae-Fagetum belongs to the subass. Euonymo verrucosae-Fagetum corydaletosum solidae Onyshchenko 2009. According to Art. 5 "on or after 1.1.2002 the type of the name of the subass. typicum must be the type of the association name".

Euonymo verrucosae-Fagetum sylvaticae typicum Onyshchenko ex Onyshchenko subass. nov. hoc loco. Holotypus: rel. 11 in table 38 (Onyshchenko, 2009: 9194). Synonym: Euonymo verrucosae-Fagetum typicum Onyshchenko 2009 (Art. 5).

Euonymo verrucosae-Fagetum sylvaticae staphyleetosum pinnatae Onyshchenko ex Onyshchenko subass. nov. hoc loco. Holotypus: rel. 24 in table 38 Onyshchenko, 2009: 91-94). Synonym: Euonymo verrucosae-Fagetum staphyleetosum pinnatae Onyshchenko 2009 (Art. 5).

Euonymo verrucosae-Fagetum sylvaticae corydaletosum solidae Onyshchenko ex Onyshchenko subass. nov. hoc loco. Holotypus: rel. 14 in table 39 (Onyshchenko, 2009: 95-98). Synonym: Euonymo verrucosae-Fagetum corydaletosum solidae Onyshchenko 2009 (Art. 5). 


\section{All. Dentario quinquefoliae-Fagion sylvaticae Borhidi} 1962

Lathyro aurei-Fagetum caricetosum digitatae Onyshchenko ex Onyshchenko subass. nov. hoc loco. Holotypus: rel. 20 in table 41 (Onyshchenko, 2009: 102105). Synonym: Lathyro aurei-Fagetum caricetosum digitatae Onyshchenko 2009 (Art. 5).

\section{All. Carpinion betuli Issler 1931}

Isopyro thalictroidis-Carpinetum brachypodietosum sylvatici Onyshchenko ex Onyshchenko subass. nov. hoc loco. Holotypus: rel. 8 in table 51 (Onyshchenko, 2009: 36-140). Synonym: Isopyro thalictroidis-Carpinetum brachypodietosum sylvatici Onyshchenko 2009 (Art. 5).

All. Querco roboris-Tilion cordatae Solomeshch et Laivin, ex Bulokhov et Solomeshch 2015

Mercurialo perennis-Quercetum roboris calamagrostietosum arundinaceae Onyshchenko ex Onyshchenko subass. nov. hoc loco. Holotypus: rel. 4 in table 58 (Onyshchenko, 2009: 161-163). Synonym: Mercurialo perennis-Quercetum roboris calamagrostietosum arundinaceae Onyshchenko 2009 (Art. 4, 5). The name of the association was validly published in 2015 (Bulokhov, Semenishchenkov, 2015).

All. Scillo sibericae-Quercion roboris Onyshchenko ex Onyshchenko all. nov. hoc loco. Holotypus: Stellario holosteae-Aceretum platanoidis Bajrak 1996. Synonym: Scillo sibericae-Quercion roboris Onyshchenko 2009 (Art. 5).

Stellario holosteae-Aceretum platanoidis caricetosum pilosae Onyshchenko ex Onyshchenko subass. nov. hoc loco. Holotypus: rel. 22 in table 59 (Onyshchenko, 2009: 165-168). Synonym: Stellario holosteae-Aceretum platanoidis caricetosum pilosae Onyshchenko 2009 (Art. 5).

Tulipo quercetorum-Quercetum roboris Onyshchenko ex Onyshchenko ass. nov. hoc loco. Holotypus: rel. 11 in table 61 (Onyshchenko, 2009: 171-173). Synonym: Tulipo quercetorum-Quercetum roboris Onyshchenko 2009 (Art. 5).

Anthrisco nitidi-Aceretum pseudoplatani Ralo et Onyshchenko ex Onyshchenko ass. nov. hoc loco. Holotypus: rel. 5 in table 1 (Ralo et Onyshchenko, 2008). This relevé had also been published in Onyshchenko (2009: 189-193) as rel. 5 in table 69. Synonym: Anthrisco nitidi-Aceretum pseudoplatani Ralo et Onyshchenko 2009 (Art. 5).

\section{REFERENCES}

Bulokhov A.D., Semenishchenkov Yu.A. Bull. Bryansk. otdel. $R B S, 2015,5(1):$ 26-32. [Булохов А.Д., Семенищенков Ю.А. Типификация и коррекция синтаксонов лесной растительности Южного Нечерноземья России и сопредельных регионов. Бюл. Брянск. отд. РБО, 2015, 5(1): 26-32].

Onyshschenko V.A. Forests of order Fagetalia sylvaticae in Ukraine, Kyiv: Alterpress, 2009, 212 pp.

Ralo V.M., Onyshchenko V.A. Naukovyi visnyk Volynskoho natsionalnoho universytetu., Biol. nauky, 2008, 3: 239-249. [Рало В.М., Онищенко В.А. Anthrisco nitidiAceretum pseudoplatani - нова асоціація із Західного Поділля. Наук. вісн. Волинськ. нац. ун-ту., Біол. науки, 2008, 3: 239-249].

Weber H.E., Moravec J., Theurillat J.-P. International Code of Phytosociological Nomenclature. 3rd ed. J. Veget. Sci., 2000, 11: 739-768.

Рекомендує до друку Д.В. Дубина

Надійшла 12.07.2016

Онищенко В.А. Валідизація назв деяких синтаксонів порядку Fagetalia sylvaticae 3 України. Укр. бот. журн., 2017, 74(1): 35-36.

Інститут ботаніки ім. М.Г. Холодного НАН України вул. Терещенківська, 2, Київ 01004, Україна

Валідизовано назви союзу Scillo sibericae-Quercion roboris (мезофільні листопадні ліси Східної Європи у Лісостеповій і Степовій зонах на багатих грунтах), асоціацій Stellario holosteae-Fagetum, Euonymo verrucosae-Fagetum, Anthrisco nitidi-Aceretum pseudoplatani, Tulipo quercetorumQuercetum roboris i 10 субасоціацій.

Ключові слова: Fagetalia sylvaticae, Scillo sibericae-Quercion roboris, синтаксономія, синтаксон

Онищенко В.А. Валидизация названий некоторых синтаксонов порядка Fagetalia sylvaticae из Украины. Укр. бот. журн., 2017, 74(1): 35-36.

Институт ботаники им. Н.Г. Холодного НАН Украины ул. Терещенковская, 2, Киев 01004, Украина

Валидизированы названия союза Scillo sibericae-Quercion roboris (мезофильные листопадные леса Восточной Европы в Лесостепной и Степной зонах на богатых почвах), ассоциаций Stellario holosteae-Fagetum, Euonymo verrucosae-Fagetum, Anthrisco nitidi-Aceretum pseudoplatani, Tulipo quercetorum-Quercetum roboris и 10 субассоциаций.

Ключевые слова: Fagetalia sylvaticae, Scillo sibericaeQuercion roboris, синтаксономия, синтаксон 